\title{
Integration fluoreszenter Mikrosensoren in mikrofluidische Elektrophoreseplattformen mittels Tintenstrahldruck und Photopolymerisation
}

\author{
C. Herzog ${ }^{1}$, E. Beckert ${ }^{2}$, S. Nagl ${ }^{1}$ \\ ${ }^{1}$ Institut für Analytische Chemie, Universität Leipzig, Leipzig/ Deutschland, \\ nagl@chemie.uni-leipzig.de \\ ${ }^{2}$ Fraunhofer-Institut für Angewandte Optik und Feinmechanik (IOF), Jena/ Deutschland
}

\begin{abstract}
Kurzfassung:
In der vorliegenden Arbeit konnten optische, Chemosensoren erfolgreich in mikrofluidische Elektrophoreseplattformen integriert werden. Die Sensoren zeigen Änderungen von verschiedenen Umgebungsparametern wie $\mathrm{pH}$-Wert, Temperatur oder Sauerstoffgehalt durch eine Veränderung ihrer Lumineszenzintensität in mikroelektrophoretischen Trennverfahren an. Diese Eigenschaften werden im Mikrochip zur Visualisierung von chemischen Spezies genutzt. Die Immobilisierung der verwendeten Indikatoren in mikochipintegrierten Sensorschichten konnte ohne Verlust der optischen Eigenschaften durch kovalente Bindung oder Einbettung in polymere Netzwerke erreicht werden. Die Mikrofabrikation von Sensorstrukturen erfolgte zum einen mittels Tintenstrahldruck und zum anderen via Photopolymerisation. Somit können ortsaufgelöst sowohl linienförmige Sensorstrukturen oder arrays als auch planare Sensorschichten erzeugt werden. Die Integration von $\mathrm{pH}$-Sensoren in mikrofluidische Chips für freiflussisoelektrische Fokussierung erlaubt die Echtzeitüberwachung des lokalen pH-Werts im Trennbett. Damit konnte die Quantifizierung des isoelektrischen Punkts verschiedener untersuchten Analyten realisiert werden. Zur Beobachtung von Elektrolysephänomenen bzw. der Joule'schen Wärmeentwicklung während der Elektrophorese wurden mikrochipintegrierte Sauerstoffsensoren bzw. Temperatursensoren entwickelt. Solche multifunktionellen mikrofluidischen Chips ermöglichen die Kombination von Trennverfahren mit integrierter Überwachung von Umgebungsparametern.
\end{abstract}

Schlüsselwörter: integrierte Mikrosensoren, mikrofluidische Freiflusselektrophorese, optische Chemosensoren, isoelektrische Fokussierung

\section{Einleitung}

Die Miniaturisierung chemischer Analysensysteme bietet einzigartige Vorteile wie höhere Effizienz, Sicherheit, Portabilität und Umweltfreundlichkeit. Die mikrofluidische Freiflusselektrophorese ( $\mu \mathrm{FFE})$ ist eine milde Trennmethode für biologische und chemische Spezies und ermöglicht die kontinuierliche Auftrennung von Gemischen, auch im präparativen Maßstab. Jedoch müssen zur Verfolgung von Analyten und Umgebungsparametern meist externe Marker oder indirekte, off-line Methoden angewendet werden. Durch Integration von optischen, Chemosensoren in solche Systeme soll die onchip Überwachung von mehreren Prozessparametern simultan zur Auftrennung ermöglichen. Dazu müssen allerdings die Sensormatrizen präzise, ortsaufgelöst und mit hoher Stabilität in funktionelle Mikrochipplattformen eingebracht werden, ohne dabei deren optische Eigenschaften zu verändern. In diesem Zusammenhang werden in dieser Arbeit Fabrikationsmethoden basierend auf Tintenstrahldruck und Photopolymerisation entwickelt (Abb. 1).

\section{Mikrochipfabrikation}

Die Herstellung der multifunktionellen Mikrochips basiert auf einer Kombination von Photolithographie und Tintenstrahldruck. Im ersten Schritt wird ein Temperatur- oder Sauerstoffindikator in einer polymeren Schicht immobilisiert. Dazu wird eine Lösung aus Metalloporphyrinen (z. B. Pd-TFPP) bzw. Rutheniumtrisphenantrolin in Oligoethylenglykoldiacryl und Photoinitiator auf einen Glasträger aufgebracht und unter UV-Licht auspolymerisiert (Abb. 1a). 


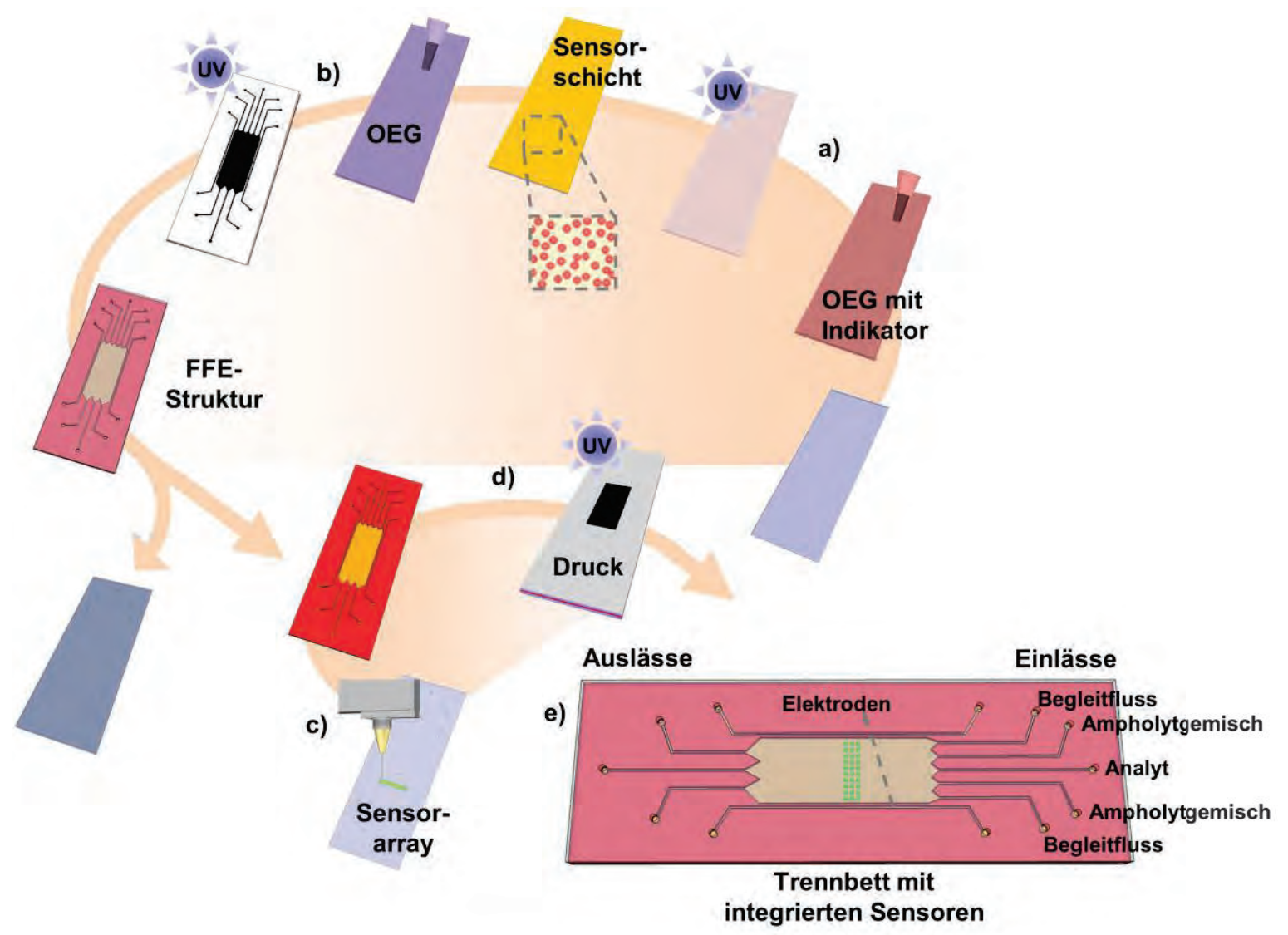

Abb. 1. Fertigungsprozess von $\mu F F E-C h i p s$ mit integrierten Mikrosensoren: (a) Photopolymerisation der Sensorschicht, (b) Photopolymerisation der $\mu F F E-S t r u k t u r, ~(c)$ Tintenstrahldruck von

Sensorstrukturen, (d) Verbindung von bedruckter Deckelplatte mit der mikrofluidischen Struktur

(e) komplettierter multifunktioneller Mikrochip.

In einem weiteren Schritt erfolgt die Fabrikation der mikrofluidischen Struktur aus Oligoethylenglykoldiacrylat mit Hilfe eines Photoinitiators und einer Photomaske unter UVBelichtung (Abb. 1b). Als letzter Schritt erfolgt die Integration der mittels Tintenstrahldruck hergestellten Sensorstruktur zur pH-WertDetektion (Abb. 1c). Dazu wird die bisherige Deckelplatte gegen einen vorbereiteten Glasträger mit dem Sensor ausgetauscht. Anschließend erfolgt erneut ein Photopolymerisationsschritt zum Verbinden der beiden Komponenten (Abb. 1d). Der Tintenstrahldruck erfolgt unter Nutzung eines automatisierten, piezoelektrischen Dosierventils. Hierbei wird eine Lösung bestehend aus einem $\mathrm{pH}$-sensitiven Fluoresceinderivat und Polyhydroxyethylmethylacrylat in einem Gemisch aus Ethanol und Wasser über eine Dosiernadel auf eine Glasoberfläche aufgebracht. Der gesamte Herstellungszyklus ist in Abb. 1 dargestellt.

Die beiden eingesetzten Techniken zur gezielten Generierung von chip-integrierten Mikrosensoren eröffnen vielfältige Applikationsmöglichkeiten.

\section{Experimenteller Aufbau}

Die Beobachtung und Detektion einer isoelektrischen Fokussierung der Analyten in einem multifunktionalen Mikrochip erfolgte an einem inversen Fluoreszenzmikroskop. Als Anregungslichtquelle wurde eine Quecksilberhochdruckdampflampe verwendet. Die verschiedenen spektralen Kanäle für die Lumineszenzanregung/-emission der untersuchten Analyten, des $\mathrm{pH}$-Sensorarrays und sauerstoff- und temperatursensorischer Schichten. (Abb. 2) Die Detektion der Lumineszenzemission erfolgt mittels einer CCD-Kamera. Über die gewählten Indikatoren und Marker kann eine spektrale Separation erreicht und somit eine simultane Detektion der einzelnen Parameter realisiert werden.

Die mikrofluidische Struktur (Abb. 1e) eines Chips für die freiflussisoelektrische Fokussierung besteht aus einem Trennbett, einem Analyteinlass und je zwei Einlässen für Ampholytgemisch sowie Begleitströme. In der gewählten Struktur besitzt der Aufbau drei Auslässe, um die getrennten Analyten auch separiert aufzufangen. Weiterhin befinden sich neben dem Trennbett ein Anoden- und ein 
Kathodenkanal mit entsprechenden Ein- und Auslässen, um das elektrische Feld einzukoppeln. Das Ampholytgemisch ermöglicht die Ausbildung eines $\mathrm{pH}-$ Gradienten im Trennbett und ist somit essentiell, um eine isoelektrische Fokussierung der Analyten zu erreichen.

a)

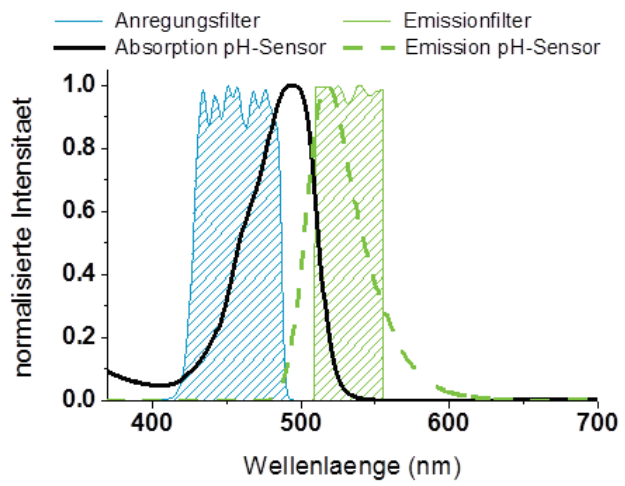

b)
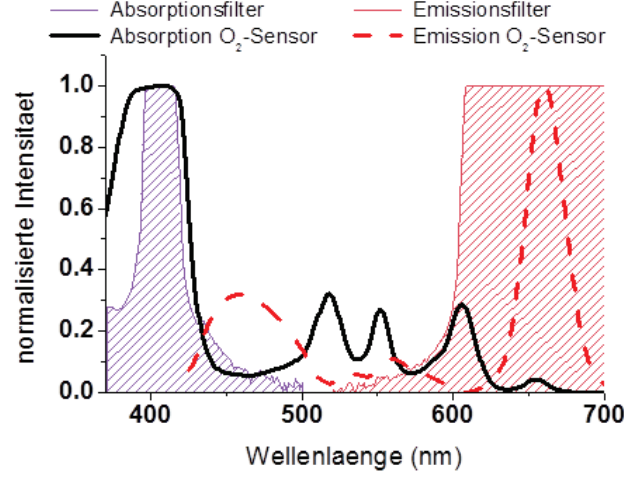

C)

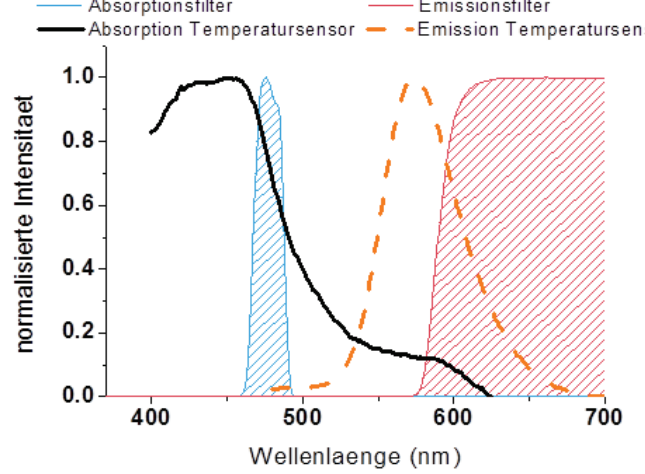

Abb. 2. Optisches Setup: Anregungs- und Emissionsspektren sowie durch Filter selektierte spektrale Kanäle der mikrochipintegrierten Sensoren. (a) $\mathrm{pH}$-Sensors, (b) Sauerstoffsensor sowie (c) Temperatursensor

\section{Charakterisierung der Sensoren}

Mittels Tintenstrahldruck konnten sowohl Sensorarrays als auch linienförmige $\mathrm{pH}$ Sensorstrukturen aus verschiedenen Materialien generiert und nachfolgend in $\mu F F E-C h i p s$ integriert werden. Mit diesem Verfahren können Sensorstrukturen mit einer Höhe von 0,4 $1,7 \mu \mathrm{m}$, welche den Fluss im Trennbett nicht signifikant stören und gute Sensitivität für $\mathrm{pH}$ Änderungen aufweisen, erzeugt werden
(Abb. 2a). Das Verfahren ermöglicht die Immobilisierung der Indikatoren und die Integration von Sensorstrukturen in Mikrochips ohne den Verlust der Eigenschaften des $\mathrm{pH}$-sensitiven Farbstoffes. Dies wird anhand der Kalibrationskurve in Abb. 3 deutlich. Die Fluoreszenzintensität der Marker zeigt sowohl vor als auch nach der Integration in einen mikrofluidischen Chip eine starke Abhängigkeit vom pH-Wert (Abb. 3).

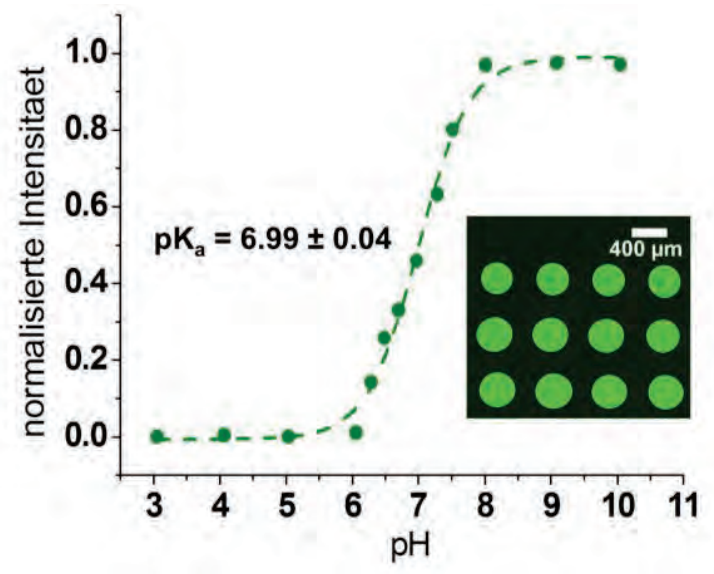

Abb. 3. pH-Abhängigkeit der Fluoreszenz eines mittels Tintenstrahlduck hergestellten chipintegrierten pH-Mikrosensors (Fluoresceinisothiocyanat kovalant gebunden an Polyhydroxyethylmethacrylat), rechts: Falschfarbenfluoreszenzbild eines Ausschnitts des Sensorarrays)

Weiterhin weist ein solcher Sensor ein schnelles Ansprechen auf eine Änderung des lokalen $\mathrm{pH}-$ Werts von wenigen Sekunden bis Sekundenbruchteilen auf. Die Ansprechzeit $t_{95}$ beträgt für die Änderung von basisch (Britton Robinson Puffer (BRP), pH 10) zu sauer (BRP pH 3) 0,27 $\pm 0,06 s$ und 1,62 $\pm 0,19 s$ von sauer zu basisch. Dies erlaubt die $\mathrm{pH}$ Beobachtung im Chip ohne nennenswerte Verzögerung.

Flächige Temperatur- und Sauerstoffsensoren können mittels Photopolymerisation von entsprechenden Indikatoren in acrylierten Oligoethylenglykolen (OEG) hergestellt werden (Abb. 1a).

Die Abhängigkeit der Lumineszenzintensität eines Sauerstoffschichtsensors basierend auf einem Metalloporphyrin wurde untersucht und konnte mittels eines two-site-model-fits ausgewertet werden (Abb. 4). Die Nachweisgrenze (LOD 99 : $1 \%$ der Ausgangsphosphoreszenz gelöscht) konnte mit $0,0772 \mathrm{~g} / \mathrm{L} \mathrm{O}_{2}$ ermittelt werden, was auf eine gute Sensitivität des Mikrosensor hinweist. Diese Sensorschichten sollen die Untersuchung der Elektrolyse während einer freiflusselektrophoretischen Trennung ermöglichen. 


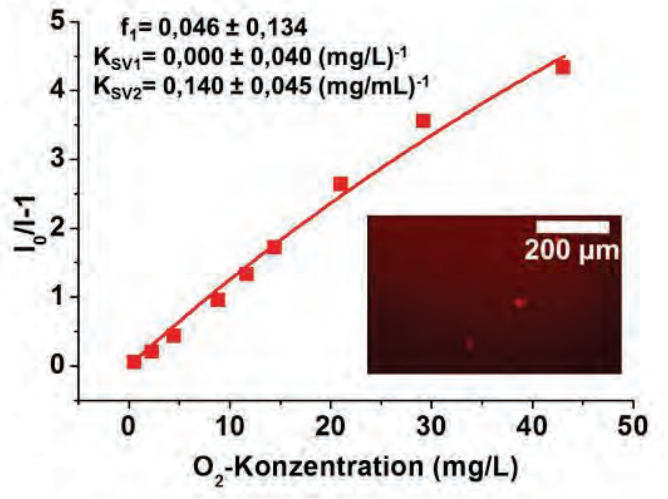

Fig. 4. Sauerstoffsensitivität einer photopolymerisierten Schicht von Palladium mesotetrapentafluorophenylporpyhrin in Oligoethylenglyokoldiacrylat (Ausschnitt: Falschfarbenfluoreszenzbild der Schicht)

Ein Temperatursensor konnte ebenfalls erfolgreich als Schicht generiert werden. Dazu wurde der Indikator (Ruthenium-tris-1,10phenanthrolin) durch Umhüllung mit Polyacrylnitril (PAN) in nanopartikuläre Form gebracht. Dies minimiert Kreuzsensitivitäten durch Sauerstoff. Die Nanopartikel wurden, wie im Kapitel Mikrochipfabrikation beschrieben, in einem Oligomer photopolymerisiert. Aus dem unten dargestellten Diagramm (Abb. 5) und einem ermittelten $\mathrm{LOD}_{99}$ von $0,89^{\circ} \mathrm{C}$ wird die prägnante Temperaturabhängigkeit der Lumineszenzintensität des Indikators mit steigender Temperatur deutlich. Die Integration eines solchen Sensors in miniaturisierte Elektrophoreseplattformen ermöglicht das Monitoring der Joule'schen Wärmeentwicklung, welche die analytische Stofftrennung während der Elektrophorese negativ beeinflusst.

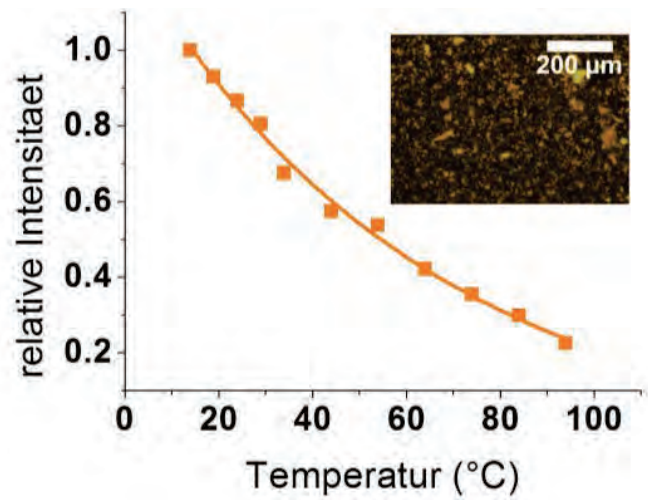

Abb. 5. Temperatursensitivität einer photopolymerisierten Schicht von Ruthenium-tris-1,10phenanthrolin in PAN in Oligoethylenglyokoldiacrylat (Ausschnitt: Falschfarbenfluoreszenzbild der Schicht)

\section{Isoelektrische Fokussierung}

Die generierten mikrofluidischen Freiflusselektrophorese-Plattformen mit sensorischer Funktion wurden zur isoelektrischen
Fokussierung (IEF) von Proteinen verwendet. Die Proteine können im Mikromaßstab anhand ihres isoelektrischen Punkts (IP) aufgetrennt und der IP mittels des chip-integrierten $\mathrm{pH}$ Sensors online quantifiziert werden (Abb. 6). Hier wurden die mit Chromeo P503 markierten Proteine Rinderserumalbumin (BSA), Conalbumin und Chymotrypsin aufgetrennt und ihre IPs in einem anderen Spektralbereich mithilfe des integrierten $\mathrm{pH}$ Sensorarrays erfasst. Es ergaben sich IP von 5,17 für BSA; 5,99 für Conalbumin und 7,71 für Chymotrypsin mit nur geringen Abweichungen von maximal 0,59 zu den aus der Literatur bekannten Werten.

a)
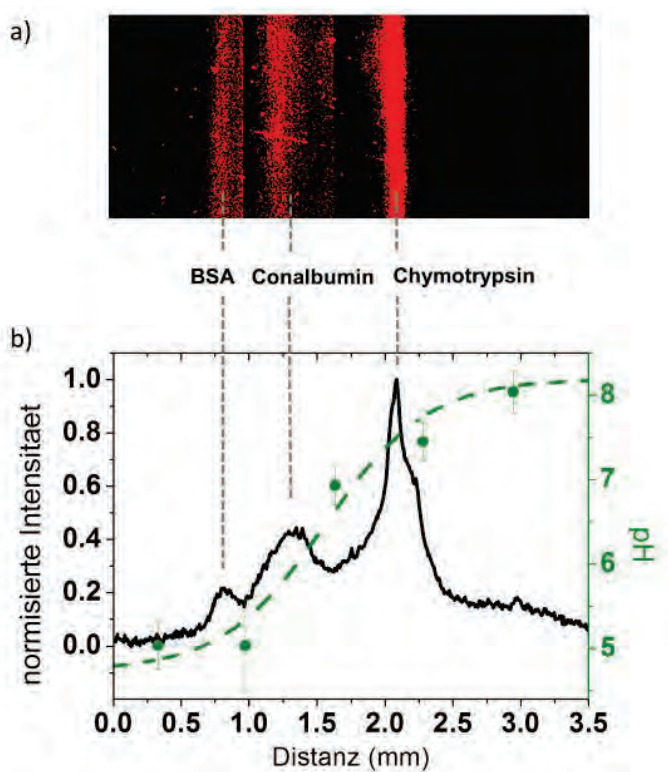

Abb. 6. a) Falschfarbenfluoreszenzbild der freiflussisoelektrischen Fokussierung von fluoreszenzmarkierten Proteinen in einem multifunktionellen Mikrochip mit integrierter $\mathrm{pH}$ Überwachung. b) Darstellung der Trennung (schwarz) sowie dem von einem $\mathrm{pH}$-Sensorarray angezeigten $\mathrm{pH}$-Gradienten (grün).

\section{Zusammenfassung}

Die Integration von Sensorstrukturen in mikrofluidische Elektrophoresechips konnte durch zwei verschiedenen Methoden gezeigt werden. Zum einen ist mittels Tintenstrahldruck gelungen, eine ortsaufgelöste Immobilisierung eines $\mathrm{pH}$-Sensors im Mikrochip gelungen. Darüber hinaus konnte auch die Generierung von Sensorschichten für Temperatur- und Sauerstoffmonitoring mittels Photopolymerisation realisiert werden.

Diese mikrochipintegrierten Sensoren erlauben eine räumlich aufgelöste Echtzeitüberwachung von analytisch bedeutsamen Prozessparametern und sollen zur Überwachung sowie Optimierung von miniaturisierten elektrophoretischen Trennprozessen verwendet werden. 